\title{
The Language Hoax. Why the World Looks the SAME IN ANY LANGUAGE
}

\section{César Cisternas Irarrázabal*}

El título de esta obra, que se traduce como "El engaño del lenguaje. Por qué el mundo se ve igual en cualquier lenguaje", adelanta de manera precisa el problema que aborda. En efecto, a lo largo de sus páginas se somete a una rigurosa crítica la hipótesis denominada determinismo lingüístico, whorfianismo o hipótesis Sapir-Whorf, la cual ha llegado a ser prácticamente un axioma en distintos trabajos emprendidos desde diversas disciplinas en el ámbito de la cultura. La hipótesis en cuestión señala que cada lengua moldea el pensamiento de sus hablantes repercutiendo en su cosmovisión. Tal como lo indica Yule (2010) se han escrito críticas y se ha presentado evidencia en contra de ésta. No obstante, McWhorter lleva a cabo su tarea no desde la mera ensayística, sino que desde una amplia base de estudios empíricos realizados con hablantes de distintas lenguas reforzada con análisis comparados de distintas sistemas lingüísticos, lo que le entrega un altísimo grado de rigurosidad.

El libro se divide en seis capítulos más una introducción. En la introducción se plantea el objetivo del trabajo, aclarando que su planteamiento no niega la relación entre lengua y cultura, sino que sólo pretende echar abajo la asunción del whorfianismo.

En el primer capítulo McWhorter expone la evidencia que los whorfianistas presentan como respaldo a su postura. En este punto el autor pone especial atención a aquellos estudios recogidos por Deutscher (2010), principalmente referentes a los colores, el género y las referencias espaciales. En gran medida el trabajo de McWhorter es una respuesta a tal libro -basta apreciar los subtítulos de ambas obras-. El primer caso discutido es un estudio cuyos resultados indican que existe una relación entre la forma en la que la lengua hablada expresa un lapso grande de tiempo -sea como distancia o como cantidad-y la capacidad para 
predecir cuánto demorarán ciertos eventos en ocurrir. En segundo lugar analiza otro estudio que indica que los rusoparlantes distinguen más rápidamente distintas tonalidades de azul que un hablante de inglés por tener palabras diferentes para azul y celeste. Sin embargo, las diferencias en ambos estudios entre los más veloces y los más tardos son tan reducidas como unas decenas de milisegundos. Ante esto, McWhorter es enfático en señalar que estos antecedentes no pueden ser vistos como evidencia de formas de pensar divergentes que traerían como efecto cosmovisiones particulares como lo platea el whorfianismo.

Otras supuestas evidencias que presenta el whorfianismo para sustentarse contemplan la afirmación de que un pueblo amazónico cuya lengua no consta de números es incapaz de realizar cálculos matemáticos, que otro grupo africano es incapaz de distinguir el verde del azul pues su lengua contempla sólo un término para ambos, etc. Todas sagazmente echadas abajo y ridiculizadas brillantemente por McWhorter. Hacia el final del capítulo, el autor hace su punto: las culturas humanas divergen enormemente y las lenguas reflejan las culturas, lo que éstas no hacen es moldear el pensamiento, ni la cosmovisión, por sí solas. De este modo, al término esta sección del libro quedan formulados dos cargos contra la mencionada corriente lingüística: 1) extrapolación injustificada de resultados deexperimentos lingüísticos, y 2) conclusiones grandilocuentes erradas a partir del estudio de ciertos grupos y sus lenguas.

El segundo capítulo introduce la problemática de la evolución de las lenguas. John McWhorter llega a este tópico puesto que sostiene que uno de los factores que le da credibilidad a la tesis de que las lenguas modelan el pensamiento, es la idea de que las lenguas reflejan el cómo son sus hablantes. En esta empresa el autor analiza el papel que juega la necesidad de los hablantes en el desarrollo de las lenguas. Tras un recorrido que incluye ciertas palabras, marcas evidenciales y tiempos verbales, McWhorter deja en claro que la necesidad de quienes hablan una lengua no tiene mayor incidencia -salvo la creación de algunas nuevas palabras para nombrar nuevas cosas- sobre la evolución de sus lenguas. A esto se suma un punto sobre el que volverá en el capítulo quinto, a saber, el hecho de que las lenguas se conforman mucho antes que las culturas presentes de los grupos; es decir, son una herencia antes que una creación. Así, concluye que: "Las lenguas se desarrollan a través de desvíos que operan debajo del nivel de la conciencia, y esto no es una 
opinión, sino un hecho, fundamental en cualquier clase introductoria sobre el cambio lingüístico" (p. 56).

En el tercer capítulo McWhorter aclara que él sólo imputa la relación entre lengua y cultura que plantea el whorfianismo, profundizando los puntos de encuentro que efectivamente existen entre ambas. Indica que es innegable que el uso de diferentes palabras o grupos de palabras que aparentemente poseen el mismo significado varía según la cosmovisión de los hablantes (lo que es estudiado por la etnosemántica). Asimismo ejemplifica cómo la lengua es utilizada de formas diferentes en las prácticas culturales de distintos grupos humanos (campo de la etnografía de la comunicación). Por otra parte, expone que el éxito la hipótesis Sapir-Whorf dentro del campo de la lingüística se debe principalmente al deseo de oponerse a la teoría de una gramática universal de Noam Chomsky y sus seguidores, indicando que la tesis de su libro no implica necesariamente adhesión a la propuesta de Chomsky.

El cuarto capítulo centra su atención en el hecho de que las lenguas difieren en cuanto a qué incluyen en las oraciones. Recorriendo el inglés, el Atsugewi (idioma nativo americano), el ruso, el hebreo, el tagalog y el chino mandarín, el autor revela el lado más peligroso del whorfianismo: jerarquizar los niveles intelectuales de distintas comunidades lingüísticas. Esto ha sucedido en un estudio que sugirió que el hablante de chino mandarín -lengua de muy baja complejidad gramatical- tienen más dificultades al reconocer lo hipotético de lo real -al no constar con marcadores de condicionalidad-. McWhorter describe lo vehemente que ha sido la oposición a las conclusiones a este tipo de investigaciones, ilustrando la contradicción que existe en el campo de la lingüística respecto al determinismo lingüístico: es aceptado visceralmente cuando se habla de ventajas (como la capacidad de distinguir una gama más amplia de colores), pero es objetada en casos como el anteriormente descrito.

El quinto capítulo es un ejercicio interesante por intentar trasladar el foco del whorfianismo desde las lenguas exteriores al mundo anglo hacia el inglés. Tal empeño se encuentra guiado por la pregunta ¿cuál es la cosmovisión de la lengua inglesa? Tomando como caso una frase común de un adolescente afroamericano, socava de forma perspicaz los argumentos de la mencionada corriente lingüística. A lo largo de las páginas de esta sección se recalca que los matices de la vida que una 
lengua marca preferentemente, no son un tema relativo a la necesidad de los hablantes o la naturaleza de sus hablantes, sino que materia de mera casualidad. Se plantea que si bien es cierto que las lenguas incorporan palabras cuando los sujetos requieren nombrar nuevas cosas, postular que éstas evolucionan de acuerdo a la necesidad de los individuos es insostenible.

Asimismo en este capítulo se revela otro punto débil del whorfianismo. Esta corriente teórica plantea que la lengua modela el pensamiento, pero es un hecho conocido que las lenguas se conforman mucho antes que la cultura actual de sus hablantes. Entonces, el whorfianismo debería responder qué estadio de la lengua es el que moldea el pensamiento de la comunidad lingüística. Bajo esta línea argumentativa, quedaría por esclarecer, por ejemplo, cuál es la lengua que ha tenido incidencia en el pensamiento de los angloparlantes: ¿el antiguo inglés o el inglés moderno? Además, restaría la interrogante de qué papel juegan las diferentes variantes de una lengua en sus hablantes.

El sexto capítulo cumple las funciones de una conclusión recopilatoria. En este el autor plantea que más que científica la vocación del whorfianismo es de abogacía, buscando representar a las culturas indígenas y minoritarias como legítimas y sofisticadas. En este curso de acción, cae en el error de darle mayor importancia a lo particular que a lo universal. Asimismo, resume sus cuestionamientos al determinismo lingüístico en tres puntos:

1) Su doble estándar: si se desea seguir la tesis del whorfianismo se debe aplicar sus principios por igual a todos los grupos. Esto implicaría reconocer, por ejemplo, si se plantea que las lenguas en las que el género masculino es el predeterminado son sexistas, que también existen lenguas indígenas sexistas.

2) Está marcado por un fuerte paternalismo, una profunda animadversión hacia los grupos con poder y ensalzamiento antojadizo de los grupos que carecen de éste. Se sugiere que los hablantes de lenguas mayoritarias sólo hablan, mientras que aquellos de lenguas indígenas dan lecciones de filosofía. Pecando además de presentar al otro como más diferente de lo que en realidad es. Esfuerzo en el cual se aparenta valorar a los indígenas por considerarlos más auténticos, lo que oculta la implicancia de rotularlos como atrasados. 
3) Es un producto de un estadio inmaduro de la lingüística, es insostenible en el contexto del actual desarrollo de la ciencia. Por esto mismo, y por el escaso conocimiento del público, es necesario divulgar el conocimiento en esta área.

El autor indica que sólo en un contexto de totalitarismo absoluto es imaginable que la lengua conduzca la cultura. De este modo, concluye que la diversidad lingüística es resultado de variaciones de una misma forma de pensar: la humana. Sin embargo, recalca que no persigue desmentir la relación entre cultura y lengua, lo que sería un despropósito; sino que busca refutar únicamente la relación lengua-cultura que defiende el whorfianismo.

En síntesis, a lo largo del libro se devela que el whorfianismo, a pesar de sus buenas intenciones, carece en absoluto de sustento científico, además de promover una imagen del otro similar a lo que Baudrillard (1991) llamó exotismo radical. A la vez que propugna una actitud ante el otro que resulta ser paternalista, esencialista y etnocéntrica, como Taylor (2009) describe aquella postura que defiende la igualdad de las culturas, en lugar de darles la oportunidad de someterse a una evaluación crítica y ser respetadas sólo por el gran mérito de ser culturas diferentes. De esta manera, el libro entrega sustento a la declaración que hiciera, de forma más bien intuitiva, Sarzuri-Lima (2012) respecto a que si lenguas distintas repercutieran en formas de pensar y ver el mundo diferentes, la comunicación intercultural sería una utopía sin sentido. Todos estos vicios son los que, a juicio de McWhorter, vuelven al whorfianismo aparte de no-científico-incluso peligroso.

Si bien es cierto que The Language Hoax ha sido escrito por un lingüista, el libro resulta accesible a cualquier cientista social. Esta interesante y rigurosa obra resulta especialmente importante para lingüistas, antropólogos y sociólogos dedicados a la investigación y reflexión en torno a las relaciones interculturales. 


\section{Referencias}

Baudrillard, J. (1991). La transparencia del mal. Ensayo sobre los fenómenos extremos. Barcelona: Anagrama.

Deutscher, G. (2010). Through the language glass. Why the world looks different in other languages. New York: Metropolitan Books.

Sarzuri-Lima, M. (2012). De la palabra al texto: colonialidad lingüística y luchas interculturales. Revista Integra Educativa, $5(1)$.

Taylor, C. (2009). El multiculturalismo y la política del reconocimiento (2da Ed.). México D.F.: Fondo de Cultura Económica.

Yule, G. (2010). The study of language (4ta Ed.). Cambridge: Cambridge University Press. 